\title{
The physiology and ecological implications of efficient growth
}

\author{
Benjamin RK Roller ${ }^{1,2,3}$ and Thomas M Schmidt ${ }^{3,4,5}$ \\ ${ }^{1}$ Department of Microbiology and Molecular Genetics, East Lansing, MI, USA; ${ }^{2}$ Ecology, Evolutionary Biology \\ and Behavior Program, Michigan State University, East Lansing, MI, USA; ${ }^{3}$ Departments of Internal Medicine, \\ University of Michigan, Ann Arbor, MI, USA; ${ }^{4}$ Ecology and Evolutionary Biology, Ann Arbor, MI, USA and \\ ${ }^{5}$ Microbiology and Immunology, Ann Arbor, MI, USA
}

\begin{abstract}
The natural habitats of microbes are typically spatially structured with limited resources, so opportunities for unconstrained, balanced growth are rare. In these habitats, selection should favor microbes that are able to use resources most efficiently, that is, microbes that produce the most progeny per unit of resource consumed. On the basis of this assertion, we propose that selection for efficiency is a primary driver of the composition of microbial communities. In this article, we review how the quality and quantity of resources influence the efficiency of heterotrophic growth. A conceptual model proposing innate differences in growth efficiency between oligotrophic and copiotrophic microbes is also provided. We conclude that elucidation of the mechanisms underlying efficient growth will enhance our understanding of the selective pressures shaping microbes and will improve our capacity to manage microbial communities effectively.
\end{abstract}

The ISME Journal (2015) 9, 1481-1487; doi:10.1038/ismej.2014.235; published online 9 January 2015

\section{Introduction}

The conceptual foundation of microbial physiology was built on studies of microbes during balanced growth in homogenous cultures. Schaechter et al. (1958) first established that bacteria adjust their macromolecular composition to match growth rate. The rate of a population's exponential growth is an integrated signal composed of the chemical and physical features of an environment. As stated elegantly by Neidhardt (1999), '... when growth is the ultimate interest, one cannot long delve into single enzymes and genes, or even individual pathways and mechanisms, without at some point returning to the whole cell and asking about the coordinated operation of processes.'

The growth rate of a microbe-the number of progeny produced per unit time-is an important component of fitness in most environments and therefore a pivotal life history trait. Any trait that impacts fitness by altering a microbe's reproduction or survival is termed a life history trait, and these include responses to varying resource availability, population density and other extracellular factors (Vasi et al., 1994). The collection of life history traits

Correspondence: T Schmidt, Ecology and Evolutionary Biology, University of Michigan, 1150 West Medical Center Drive, Ann Arbor 48109-5666, MI, USA.

E-mail: schmidti@umich.edu

Received 14 June 2014; revised 18 September 2014; accepted 31 October 2014; published online 9 January 2015 define a microbe's life history-the overall pattern of reproduction and survival.

A less obvious, but equally important life history trait is the efficiency of microbial growth-the number of progeny produced per unit of resource consumed. Like growth rate, growth efficiency integrates a microbe's physiology, ecology and evolutionary history. The efficiency of growth for any given microbe depends on multiple environmental and population-specific factors, including the free energy available from a resource (Linton and Stephenson, 1978), pathways for resource utilization (Flamholz et al., 2013), the availability of precursors for biomass synthesis (Stouthamer, 1973) and the fraction of available energy devoted to maintenance functions instead of growth (Hoehler and Jørgensen, 2013). We do not yet know the collection of specific genetic determinants that underlie growth efficiency, but as described below, it is obvious that efficiency is a life history trait and is under selection in most environments. Although our ultimate goal is to understand all elements of a microbes fitness in concert, including both reproduction and survival components, our primary focus in this work is on two life history traits that impact reproduction-the rate and efficiency of population growth.

Growth efficiency is important from an evolutionary perspective and has repercussions for understanding how ecosystems function. Carbon use efficiency (CUE), the amount of carbon incorporated into biomass per carbon resource consumed, is one 
way to measure growth efficiency and is a proxy for the number of progeny produced per unit resource. It also provides a quantitative measure of the impact that microbes have on nutrient cycling in an ecosystem. In animals' digestive tracts, microbes impact many essential processes for the holoorganism, especially the nutritional value extracted from their diets (McFall-Ngai et al., 2013) where microbial CUE is likely a critical variable. Heterotrophic microbes are major contributors to the global carbon cycle (Cho and Azam, 1988; Singh et al., 2010)—respiring 60 gigatonnes of terrestrial organic matter to carbon dioxide $\left(\mathrm{CO}_{2}\right)$ each year, roughly six times more than annual anthropogenic emissions (Trivedi et al., 2013) — yet we are just beginning to understand the efficiency of carbon use by heterotrophic microbes and its impact on ecosystem carbon cycling (Manzoni et al., 2012; Lee and Schmidt, 2014). Exploring variations in CUE among diverse microbes will improve our knowledge of how microbial communities impact carbon flux, from the small scale of host-microbiome interactions to largescale annual $\mathrm{CO}_{2}$ flux from an ecosystem.

We address two primary questions in this perspective: which environmental characteristics favor efficiency and what is the extent of plasticity in growth efficiency of individual microbes? Key findings are illustrated using aerobic heterotrophs, but should also apply to fermentative microbes and those that respire any of an array of terminal electron acceptors other than oxygen $\left(\mathrm{O}_{2}\right)$. In regards to the terminology used to describe efficiency, ecologists often measure growth efficiency in carbon (C) units, that is, moles of $\mathrm{C}$ incorporated into biomass per mole of $\mathrm{C}$ consumed, and use the terms CUE, microbial growth efficiency and bacterial growth efficiency interchangeably to describe this measure. Microbial physiologists and engineers more often describe efficiency in terms of yield. Yields are expressed in units that are not as easily compared across microbial populations or growth conditions, for example, biomass per gram of resource, per mole ATP or per mole of electrons. We have elected to use CUE as a measure of efficiency in addition to progeny per resource measurements. CUE varies between 0 and 1 and provides an intuitive comparison across organisms and resources.

\section{When is efficient growth favored?}

An intriguing study of how spatial heterogeneity and varying resource availability influences selection on growth rate and growth efficiency was conducted using mathematical simulations of heterotrophic microbes. In these simulations, two types of 'organisms' competed across gradients of spatial structure and resource flux. One was a rapidly growing, inefficient, respiro-fermentative organism. The other was an efficient, but slow growing, obligately respiring organism. Efficient growth was favored over rapid growth when the flux of resources was low and spatial heterogeneity was high. As the flux of resources increased and the environment became more homogeneous, the rapidly growing organism was favored (Pfeiffer et al., 2001). Potential tradeoffs between growth rate and growth efficiency have also been evaluated experimentally with genetically modified yeast strains. The competing strains were isogenic except for a single mutation that made one strain capable of using only the more efficient process of respiration but slowed growth rate. The other strain gained energy primarily through the less efficient process of fermentation and grew more rapidly (Maclean and Gudelj, 2006). When these strains competed in a homogenous, continuous culture, the rapid growing organism was more fit and outcompeted the efficient organism. Altering only the temporal availability of resources by using batch culture or in combination with spatial structure by using a metapopulation of batch cultures, allowed for the coexistence of rapid and efficient strains. Taken together, these studies indicate that a few key factors-low resource concentrations, spatial heterogeneity and temporal resource dynamics-can increase the fitness of efficient strains.

Typical laboratory cultivation differs from the conditions microorganisms experience in their natural environments, where spatial heterogeneity is pervasive (Stocker, 2012). The lack of spatial or temporal structure in typical laboratory cultivation causes resources to be a global commodity shared by the entire experimental population. Selection therefore favors rapid growth in both batch and continuous cultivation evolution experiments (Dykhuizen and Hartl, 1981; Vasi et al., 1994). Spatial heterogeneity can be accomplished in a laboratory setting by using an oil emulsion that compartmentalizes individual microbes. In one study, a population of randomly mutagenized Lactococcus lactis was serially propagated in an oil emulsion. This led to a rateefficiency tradeoff between isolated clones. Isolates with increased growth efficiency relative to the parental strain of L. lactis were observed, yet they typically grew slower than inefficient clones in the population. The clone with the greatest yield and slowest growth rate had a large increase in relative abundance throughout the 28 days of propagation, demonstrating selection favoring efficient growth (Bachmann et al., 2013).

Spatial heterogeneity, low resource concentrations and temporal resource dynamics can all favor efficient growth because each of these factors influence the scale of competition, effectively privatizing resources to individuals and shifting the cost of inefficient resource use from the community to the individual. Populations founded by inefficient organisms will be smaller than those founded by efficient organisms, given the same amount of resource for each population (Pfeiffer 
et al., 2001). Competition between organisms capable of achieving varied population sizes in spatially structured environments can lead to counter-intuitive results. Using genetically modified strains of Escherichia coli, Chuang et al. (2009) showed that selection could favor a strain that produced a larger final population size in a spatially structured metapopulation, even when it was at a growth rate disadvantage, a phenomenon that has been described statistically as the Yule-Simpson effect. This phenomenon demonstrates that considering the environmental context in which selection acts on microorganisms is critical.

Two key variables, resource availability and the free energy content of these resources, have large impacts on the physiology and growth efficiency of microbial populations and deserve a more detailed discussion and analysis. The framework presented for how these variables impact growth efficiency will then be used to develop a model of growth efficiency that distinguishes two distinct life histories.

\section{Growth efficiency varies with resource availability}

Heterotrophic bacteria utilize organic compounds for two primary purposes: as a source of energy (extracted through catabolism) and as a source of carbon molecules to build biomass (anabolic reactions). The fractionation of carbon between catabolism and anabolism varies within and between organisms. One factor that modulates the fractionation of carbon within an organism is growth rate. At submaximal growth rates, bacteria uncouple anabolism from catabolism (Tempest and Neijssel, 1984) and a larger fraction of the cell's energy budget is devoted to maintenance functions rather than biomass synthesis (Tempest and Neijssel, 1984; Russell and Cook, 1995). The decreased proportion of biosynthesis in the energy budget at submaximal growth rates can be measured by tracking the fate of carbon to biomass or $\mathrm{CO}_{2}$.

Previously published measures of the fractionation of carbon between catabolism and anabolism are often reported as biomass yields using mass units. Because yields are resource dependent, comparing yields across different resources requires converting the data to a common currency. We convert growth yields to CUE-moles of carbon incorporated into biomass per total moles of carbon consumed (equation 1a). During non-fermentative growth, the total carbon utilized by heterotrophs is equivalent to carbon incorporated into biomass production plus carbon respired, and CUE can be expressed as in equation 1b. Oxygen consumption and dry biomass measurements can also be used to calculate growth efficiency, as in equation 1c, when there is a consistent carbon content in the biomass of the organism and the respiratory quotient (RQ, the ratio of $\mathrm{CO}_{2}$ produced per $\mathrm{O}_{2}$ consumed) reflects complete oxidation of the substrate. We calculated CUE, using equations $1 \mathrm{~b}$ and $\mathrm{c}$, and the steady-state concentration of the limiting resource in a series of chemostat experiments with strains of Klebsiella aerogenes (Herbert, 1976; Neijssel and Tempest, 1976). The assumptions that the biomass has a constant carbon content and the carbon source is completely oxidized are reasonable for this organism during carbon-limited growth (Neijssel and Tempest, 1975; Herbert, 1976), so we are able to quantify the impact of resource availability on CUE.

$$
\begin{aligned}
& \mathrm{CUE}=\frac{\text { Biomass } \mathrm{C}(\mathrm{mol})}{\text { Total Cutilized }(\mathrm{mol})}=\frac{(1 \mathrm{~b})}{\text { Biomass } \mathrm{C}} \\
& =\frac{(\text { Dry biomass } \times \% \mathrm{C} \text { in biomass })}{(\text { Dry biomass } \times \text { \% } \mathrm{C} \text { in biomass })+\left(\mathrm{RQ} \times \mathrm{O}_{2} \text { utilized }\right)}
\end{aligned}
$$

To calculate the concentration of the limiting resource $(R)$ we used equation 2 , where $\mathrm{R}$ is a function of the dilution rate $(D)$ and physiological properties of the organism: the maximum specific growth rate $\left(\mu_{\max }\right)$ and the concentration of the limiting resource that supports the organism's growth at half of the maximal rate $\left(K_{\mathrm{s}}\right)$.

$$
R=\frac{D \times K_{\mathrm{s}}}{\mu_{\max }-D}
$$

During glycerol-limited growth of $K$. aerogenes in a chemostat culture, a larger fraction of the total carbon consumed is assimilated into biomass as growth rate increases and a smaller proportion is required for respiration (Figure 1a). All carbon is accounted for at each steady-state growth rate of $K$. aerogenes (biomass $\mathrm{C}+$ respired $\mathrm{C}$ ), indicating that partial oxidation products of glycerol are not accumulating in the medium.

Converting the carbon metabolism data in Figure 1a into CUE (equation 1) and plotting this against the steady-state limiting resource concentration (equation 2) provides insight into the relationship between these parameters (Figure 1b). Growth efficiency increases as the limiting resource concentration is raised during glycerol-limited growth, until reaching a plateau of maximum efficiency at higher resource concentrations. The same relationship between growth efficiency and resource concentration is apparent regardless of whether growth is phosphate, sulfate or glucose limited, assuming complete carbon source oxidation (Figure 1c). $K_{\mathrm{s}}$ values for glucose (Neijssel and Tempest, 1975) and sulfate (Owens and Legan, 1987) were taken from the literature on K. aerogenes, whereas the values for phosphate (Owens and Legan, 1987) and glycerol (Neijssel and Tempest, 1975; Owens and Legan, 1987) are derived from E. coli. Although this historical data may not perfectly reflect the $K_{s}$ values realized during the original experiment, the same relationship with CUE is 
observed even when manually altering $K_{\mathrm{s}}$ values within a larger range of values of closely related organisms from the literature (data not shown). In addition, in the sulfate- and phosphate-limited cultures, the lowest concentrations of limiting resource do not always lead to complete carbon source oxidation (Neijssel and Tempest, 1975). This is based on the measurements at a single dilution rate and will not alter our interpretations and conclusions because it will lead to a lower CUE than was calculated at the lowest resource concentrations.

These results imply that carbon metabolism becomes more efficient as growth becomes less nutritionally constrained, with an organism's maximal growth efficiency reached near unconstrained, balanced growth. As discussed below, maximal CUE is also specific to the organic source being

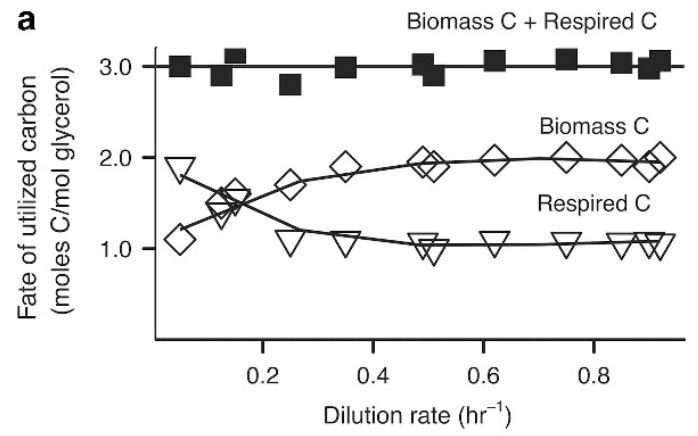

b

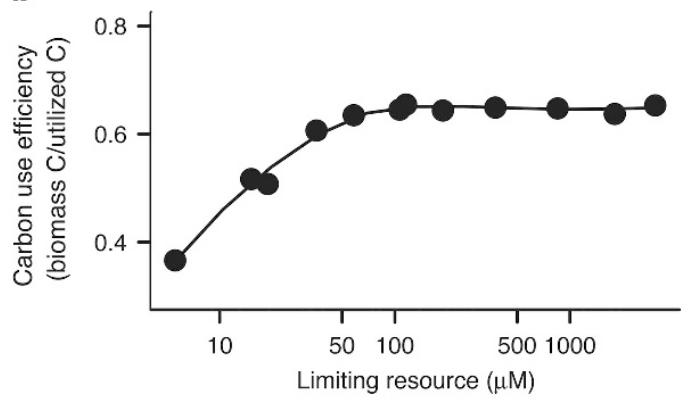

C

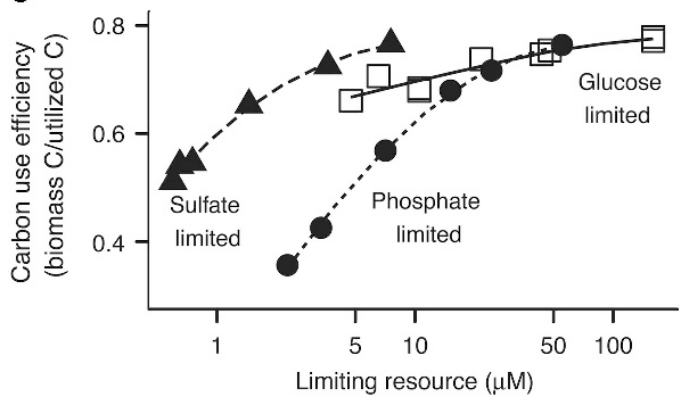

Figure 1 The influence of limiting resources on the CUE of $K$. aerogenes NCTC 418 in chemostat cultures. (a) Allocation of carbon in a glycerol-limited chemostat culture as a function of dilution rate (Herbert, 1976). (b) Variation in CUE related to the steady-state glycerol concentration (calculated from Herbert, 1976). (c) Relationship between CUE and the steady-state limiting resource concentration in glucose-, phosphate- or sulfate-limited conditions (calculated from Neijssel and Tempest, 1976). Curve fitting in all panels was generated using a locally weighted regression algorithm (LOESS) to help visualize trends. metabolized and likely specific for the entire physical and chemical environment.

\section{Growth efficiency varies with resource quality}

Growth efficiency is also dependent on the amount of energy captured during the oxidation of different organic compounds. To demonstrate the magnitude of changes in CUE due to the energy content of different carbon sources, we gathered the data from batch cultivation experiments in which 10 different species of bacteria were grown in minimal media with different organic compounds serving as the sole carbon and energy source (Linton and Stephenson, 1978). We calculated CUE assuming biomass had a constant carbon content for all organisms (Simon and Azam, 1989) and plotted against the heat of combustion per carbon atom in the organic compound supporting growth (Figure 2). Bacteria growing on resources with small amounts of free energy per carbon atom must use energy to reduce the carbon to the oxidation state of their biomass. This increased demand for energy, in the form of reducing equivalents, decreases overall CUE on low energy resources. When the energy content of the carbon in the resource and biomass (calculated from Cordier et al., 1987) is similar, the efficiency of growth stops increasing. This phenomenon has been reported for microbes in soils, as well as in pure culture (Manzoni et al., 2012).

Batch culture allows for unconstrained, balanced growth conditions and, we argue, a microbe's maximal efficiency for that environment. Yet, we still see that growth efficiency depends on the energy content of the carbon resource, even when the resource is provided in quantities that far exceed biosynthetic demand. Despite the increased variability introduced by comparing 10 different bacteria in this analysis, a strong relationship between efficiency and energy content of the carbon resource is observed.

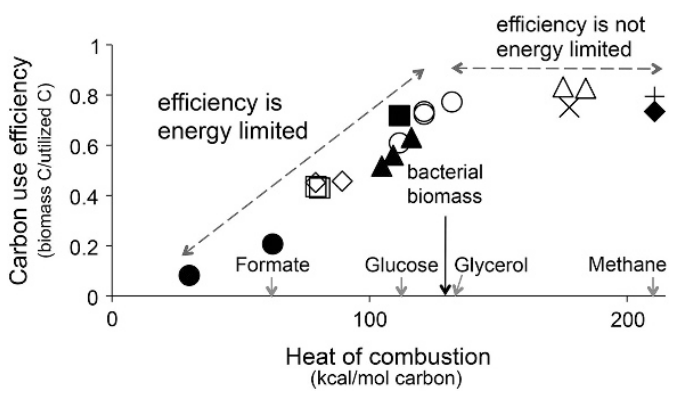

Figure 2 CUE of 10 bacterial species related to the energy content of the organic compound supporting growth (calculated from Linton and Stephenson, 1978). Distinct symbols represent different bacterial species. The heat of combustion of representative organic compounds and an average for bacterial biomass (E. coli (130.2) and M. methylotrophus (132.5), calculated from Cordier et al., 1987) are presented on the $x$ axis. 


\section{Life history and growth efficiency}

One of the best-known distinctions of microbial life histories is the copiotroph-oligotroph dichotomy. Copiotrophic microbes are selected for rapid growth when resources are abundant, whereas oligotrophic microbes have adaptations for growth in persistently resource-poor environments (Koch, 2001). This framework has many similarities to the concepts underlying $r / K$ selection theory in macroecology, where the selective pressures on organisms are a function of resource availability or population density. Unlike $r / K$ selection, the copiotroph-oligotroph dichotomy does not require differential patterns of survival or persistence.

There is a striking parallel between the conditions where oligotrophs thrive and the conditions that select for efficient organisms. Many ecosystems that have considerable oligotroph membership, such as the open ocean (Vergin et al., 2013) and bulk soil (Fierer et al., 2007), contain habitats with low resource availability and spatial structure that should select for efficient microbial growth. We propose a conceptual model that outlines how growth efficiency varies between an archetypical copiotroph and oligotroph as a function of resource concentration and quality (Figure 3).

The relationship between growth efficiency and resource concentration in our proposed model follows the same general pattern for both the oligotroph and copiotroph. The capacity for growth is indicated by the extent of the growth efficiency function, which terminates when growth is no longer supported. These two life histories have distinguishing features in the relationship between efficiency and concentration of the limiting resource in their environment. The proposed model contains the following elements and hypotheses:

1. As has been proposed previously (Zhao et al., 2013), oligotrophs are superior competitors for resources at low resource concentrations. This is visualized as the oligotroph having the capacity to grow at a much lower concentration of a limiting resource than the copiotroph.

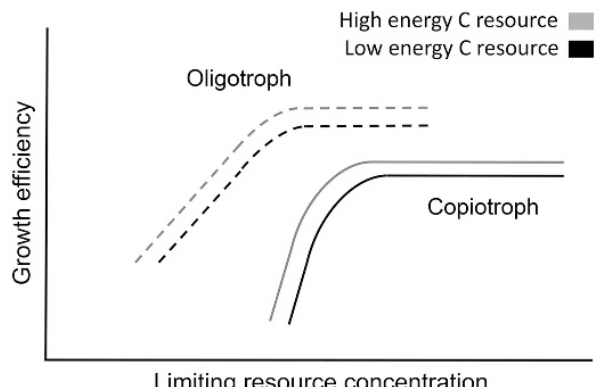

Figure 3 Proposed model of growth efficiency for distinct bacterial life histories. The efficiency of copiotrophic (solid lines) and oligotrophic (dashed lines) bacteria should be compared on resources with the same energy content (indicated by line color) and at the same limiting resource concentration.
2. On the basis of the evidence from Figure 1 , growth efficiency increases for both copiotrophs and oligotrophs as the limiting resource concentration increases up to a threshold where maximum efficiency is achieved. As a smaller proportion of carbon metabolism is directed towards maintenance energy, efficiency increases until it reaches a maximum near balanced growth.

3. We hypothesize that maintenance energy is lower for oligotrophs compared with copiotrophs. There are two consequences of this in our model: the minimum growth efficiency is higher for the oligotroph and the rate of increase in growth efficiency is slower. This extends the range of resource concentrations supporting the oligotroph's growth. Eight cellular functions have been described as the components of maintenance energy (van Bodegom, 2007) and oligotrophs have been shown to minimize costs associated with three of these functionsprotection from oxygen stress, cell motility, and the synthesis and turnover of macromolecules. A large clade of marine oligotrophs have lost the capacity to synthesize oxygen stress protectants when they are freely available in their environment (Morris et al., 2012) and many described oligotrophs are also non-motile (Lauro et al., 2009; Stocker, 2012). Additionally, genome streamlining is common in oligotrophs (Giovannoni et al., 2014), which may be an adaptation to decrease the amount of resources invested in macromolecule synthesis and turnover. Taken individually, any one of these traits is not exclusive to, or universally present in, oligotrophs. However, there is a tendency towards minimizing maintenance energy costs in oligotrophs and more work must be done to evaluate this hypothesis.

4. On the basis of the evidence from Figure 2, growth efficiency increases for both copiotroph and oligotroph as the energy content of the resource is increased. This is displayed in our model in Figure 3, as gray versus black lines.

5. We propose that the maximal growth efficiency of oligotrophs is higher than copiotrophs and that it is reached at a lower concentration of limiting resource. This is supported by evidence from one of the few comparisons of an oligotroph, Sphingopyxis alaskensis, and a copiotroph, Vibrio angustum, under identical conditions (Cavicchioli et al., 2003). The oligotroph had a greater population density at all dilution rates, and thus resource concentrations, measured in the chemostat.

6. It has been postulated that oligotrophs grow less well, or not at all, in resource-rich environments (Koch, 2001). This is captured by the termination of the oligotroph's growth efficiency functions at a lower resource concentration than the copiotroph's. 
Although many of our hypotheses are built upon observations from chemostat cultures, insufficient physiological data are available for generating hypotheses at extremely low resource concentrations-corresponding to very slow or non-growing states. Technical limitations of cultivation technology are largely responsible for this lack of data, but the physiology of extreme resource starvation, where reproduction and survival processes co-occur, likely has a large role in determining microbial fitness in natural environments. It is tempting to speculate that if oligotrophs are more efficient in all physiological states, they would have an increased carrying capacity, the parameter $K$ in $r / K$ selection, relative to copiotrophs in the same conditions. However, there are not enough data to support a universal prediction of oligotrophs possessing increased carrying capacity, persistence or other $K$-selected traits that are not directly linked to growth. We believe it is important to make predictions about growth physiology during extreme starvation, but more data are needed to understand the interplay between growth and persistence in near non-growth conditions for all life histories.

We hope these hypotheses will stimulate critical discussion of the many potential mechanisms underlying the growth efficiency of microbial populations. The physiology of an individual microbe encompasses thousands of individual reactions and growth efficiency integrates these reactions into an emergent phenotype. In addition, growth efficiency directly interacts with both ecological and evolutionary processes in microbial communities. All natural microbial environments contain spatial structure, resource limitation or temporal resource dynamics. Therefore, all natural microbial environments, from relatively stable syntrophically-associated subsurface communities to dynamic host-microbe systems, must impart some selective pressure for efficient growth on their microbial assemblages. Although the consequences of these selective pressures for ecosystem functioning are unclear, any attempts to manage microbial communities must recognize evolutionary pressures favoring efficient growth are most likely present in natural microbial systems. Just as past microbiologists have used growth rate to better understand the coordination of cellular processes necessary for reproduction, modern microbiologists have the opportunity to use growth efficiency to unify our understanding of the physiological, ecological and evolutionary processes shaping microbial communities.

\section{Conflict of Interest}

The authors declare no conflict of interest.

\section{Acknowledgements}

We would like to acknowledge Arvind Venkataraman, Byron Smith, Clive Waldron, Alex Schmidt and Zarraz Lee for valuable feedback throughout the writing process. This work was supported in part by the Department of Energy Office of Science Graduate Fellowship Program (DOE SCGF), made possible in part by the American Recovery and Reinvestment Act of 2009, administered by ORISEORAU under contract no. DE-AC05-06OR23100; the National Science Foundation's Long-Term Ecological Research Program through grant no. DEB 1027253 and the National Institutes of Health (GM0099549).

\section{References}

Bachmann H, Fischlechner M, Rabbers I, Barfa N, Branco Dos Santos F, Molenaar D et al. (2013). Availability of public goods shapes the evolution of competing metabolic strategies. Proc Natl Acad Sci USA 110: 14302-14307.

Cavicchioli R, Ostrowski M, Fegatella F, Goodchild A, Guixa-Boixereu N. (2003). Life under nutrient limitation in oligotrophic marine environments: an eco/physiological perspective of Sphingopyxis alaskensis (formerly Sphingomonas alaskensis). Microbl Ecol 45: 203-217.

Cho BC, Azam F. (1988). Major role of bacteria in biogeochemical fluxes in the ocean's interior. Nature 332: 441-443.

Chuang J, Rivoire O, Leibler S. (2009). Simpson's paradox in a synthetic microbial system. Science 323: $272-275$.

Cordier J, Butsch B, Birou B, Stockar U. (1987). The relationship between elemental composition and heat of combustion of microbial biomass. Appl Microbiol Biotechnol 25: 305-312.

Dykhuizen D, Hartl D. (1981). Evolution of competitive ability in Escherichia coli. Evolution 581-594.

Fierer N, Bradford M, Jackson R. (2007). Toward an ecological classification of soil bacteria. Ecology 88: 1354-1364.

Flamholz A, Noor E, Bar-Even A, Liebermeister W, Milo R. (2013). Glycolytic strategy as a tradeoff between energy yield and protein cost. Proc Natl Acad Sci USA 110: 10039-10044.

Giovannoni SJ, Thrash JC, Temperton Ben. (2014). Implications of streamlining theory for microbial ecology. ISME J 8: 1553-1565.

Herbert D. (1976). Stoicheiometric aspects of microbial growth. In Dean A, Ellwood DC, Evans C, Melling J (eds) Continuous Culture 6: Applications and New Fields. Ellis Horwood: Chichester, UK, pp 1-30.

Hoehler TM, Jørgensen BB. (2013). Microbial life under extreme energy limitation. Nat Rev Microbiol 11: 83-94.

Koch A. (2001). Oligotrophs versus copiotrophs. Bioessays 23: 657-661.

Lauro F, McDougald D, Thomas T, Williams T, Egan S, Rice $\mathrm{S}$ et al. (2009). The genomic basis of trophic strategy in marine bacteria. Proc Natil Acad Sci USA 106: 15527-15533. 
Lee ZM, Schmidt TM. (2014). Bacterial growth efficiency varies in soils under different land management practices. Soil Biol Biochem 69: 282-290.

Linton J, Stephenson R. (1978). A preliminary study on growth yields in relation to the carbon and energy content of various organic growth substrates. FEMS Microbiol Lett 3: 95-98.

Maclean RC, Gudelj I. (2006). Resource competition and social conflict in experimental populations of yeast. Nature 441: 498-501.

Manzoni SS, Taylor PP, Richter AA, Porporato AA, Agren GIG. (2012). Environmental and stoichiometric controls on microbial carbon-use efficiency in soils. New Phytol 196: 79-91.

McFall-Ngai M, Hadfield MG, Bosch TC, Carey HV, Domazet-Lošo T, Douglas AE et al. (2013). Animals in a bacterial world, a new imperative for the life sciences. Proc Natl Acad Sci USA 110: 3229-3236.

Morris JJ, Lenski RE, Zinser ER. (2012). The Black Queen Hypothesis: evolution of dependencies through adaptive gene loss. mBio 3: e00036-12.

Neidhardt FC. (1999). Bacterial growth: constant obsession with dN/dt. J Bacteriol 181: 7405-7408.

Neijssel O, Tempest D. (1976). Bioenergetic aspects of aerobic growth of Klebsiella aerogenes NCTC 418 in carbon-limited and carbon-sufficient chemostat culture. Arch Microbiol 107: 215-221.

Neijssel O, Tempest D. (1975). The regulation of carbohydrate metabolism in Klebsiella aerogenes NCTC 418 organisms, growing in chemostat culture. Arch Microbiol 106: 251-258.

Owens J, Legan J. (1987). Determination of the Monod substrate saturation constant for microbial growth. FEMS Microbiol Lett 46: 419-432.

Pfeiffer T, Schuster S, Bonhoeffer S. (2001). Cooperation and competition in the evolution of ATP-producing pathways. Science 292: 504-507.

Russell JB, Cook GM. (1995). Energetics of bacterial growth: balance of anabolic and catabolic reactions. Microbiol Rev 59: 48-62.
Schaechter M, Maaloe O, Kjeldgaard N. (1958) Dependency on medium and temperature of cell size and chemical composition during balanced growth of Salmonella typhimurium. Microbiology 19: 592 .

Simon M, Azam F. (1989). Protein content and protein synthesis rates of planktonic marine bacteria. Mar Ecol Prog Ser 51: 201-213.

Singh BK, Bardgett RD, Smith P, Reay DS. (2010). Microorganisms and climate change: terrestrial feedbacks and mitigation options. Nat Rev Microbiol 8 : 779-790.

Stocker R. (2012). Marine microbes see a sea of gradients. Science 338: 628-633.

Stouthamer AH. (1973). A theoretical study on the amount of ATP required for synthesis of microbial cell material. Antonie Van Leeuwenhoek 39: 545-565.

Tempest D, Neijssel O. (1984). The status of YATP and maintenance energy as biologically interpretable phenomena. Ann Rev Microbiol 38: 459-513.

Trivedi P, Anderson IC, Singh BK. (2013). Microbial modulators of soil carbon storage: integrating genomic and metabolic knowledge for global prediction. Trends Microbiol 21: 641-651.

van Bodegom P. (2007). Microbial maintenance: a critical review on its quantification. Microb Ecol 53: 513-523.

Vasi F, Travisano M, Lenski RE. (1994). Long-term experimental evolution in Escherichia coli. II. Changes in life-history traits during adaptation to a seasonal environment. Am Nat 432-456.

Vergin KL, Done B, Carlson CA, Giovannoni SJ. (2013). Spatiotemporal distributions of rare bacterioplankton populations indicate adaptive strategies in the oligotrophic ocean. Aquat Microb Ecol 71: 1-13.

Zhao Y, Temperton Ben, Thrash JC, Schwalbach MS, Vergin KL, Landry ZC et al. (2013). Abundant SAR11 viruses in the ocean. Nature 494: 357-360. 